\title{
RKKY Interaction in Gapped or Doped Graphene
}

\author{
Eugene Kogan \\ Department of Physics, Bar-Ilan University, Ramat Gan, Israel \\ Email: Eugene.Kogan@biu.ac.il
}

Received November 10, 2012; revised December 10, 2012; accepted January 8, 2013

\begin{abstract}
In our previous work [1], we calculated RKKY interaction between two magnetic impurities in pristine graphene using the Green's functions (GF) in the coordinate-imaginary time representation. Now we show that the calculations of the GF in this representation can be simplified by using the Feynman's trick, which allows to easily calculate RKKY interaction in gapped graphene. We also present calculations of the RKKY interaction in gapped or doped graphene using the coordinate-imaginary frequency representation. Both representations, corresponding to calculation of the bubble diagram in Euclidean space, have an important advantage over those corresponding to calculation in Minkowskii space, which are very briefly reviewed in the Appendix to the present work. The former, in distinction to the latter, operate only with the convergent integrals from the start to the end of the calculation.
\end{abstract}

Keywords: Graphene; RKKY Interaction; Green's Functions

\section{Introduction}

RKKY interaction between two magnetic impurities in graphene was theoretically studied quite intensely during last several years [1-19] (A terse but precise review of the issue one can find in the book by M. Katsnelson [20]). One may ask, why the problem, which is in principle so simple (in the lowest order of perturbation theory, as it was treated in all the papers referenced above, the problem is equivalent to calculation of a single bubble diagram), was the subject of so many publication, using different approaches?

One of the answers to this question is connected with the fact that a simply written integral is not necessarily a simply calculated integral. More specifically, in most of the approaches mentioned above the integrals defining the RKKY interaction in graphene turned out to be divergent, and to obtain finite values from these divergent integrals one has to implement the complicated (and to some extent arbitrary) cut-off procedure. The cure from this disease turned out to be calculation of this diagram not in Mikowskii, but in Euclidean space. Green's functions (GF) in coordinate-imaginary frequency representation were used in [3]. GF in coordinate-imaginary time representation were used in [1,7].

In the present work we generalize the approach of our previous publication [1] to treat the case of gapped graphene. Not to distract attention of the reader from the aspects of the physics we are going to concentrate upon, we consider a toy model of graphene, with free electrons being described by the $2 \mathrm{~d}$ Dirac Hamiltonian. The exis- tence of two Dirac points in graphene leads to additional angular dependent factor in the formula for the RKKY interaction. This angular dependence was thoroughly studied previously $[5,8]$ and does not interfere with the physics we are discussing in this work.

The effective exchange RKKY interaction between the two magnetic impurities with the spins $\boldsymbol{S}_{1}$ and $\boldsymbol{S}_{2}$, sitting on top of carbon atoms with the relative radius-vector $R$ is

$$
H_{\mathrm{RKKY}}=-\frac{1}{4} J^{2} \chi(R) S_{1} \cdot S_{2}
$$

where $J$ is the contact exchange interaction between each of spins and the graphene electrons, and $\chi(R)$ is the free electrons charge susceptibility, depending upon whether the carbon atoms belong to the same, or different sublattices.

\section{Imaginary Time Representation: Gapped Graphene}

The susceptibility expressed through the GF in the imaginary time-coordinate representation can be written as $[1$, $5,6]$

$$
\chi(R)=-4 \int_{0}^{\infty} G(\mathbf{R} ; \tau) G(-\mathbf{R} ;-\tau) \mathrm{d} \tau .
$$

Consider electrons in $2 \mathrm{~d}$ described by Dirac equation

$$
\left[i \partial_{t}+i v \gamma \cdot \partial-\gamma^{0} m\right] \psi=0,
$$

where $\gamma \cdot \partial=\gamma^{1} \partial_{1}+\gamma^{2} \partial_{2}$. To find the GF in coordinatetime representation it is convenient to use the trick in- 
troduced by Feynman [21], that is to represent the GF as

$$
G(R, t)=\left[i \partial_{t}+i v \gamma \cdot \partial+\gamma^{0} m\right] I(R, \tau),
$$

where

$$
I(R, t)=\int \frac{\mathrm{d}^{2} k}{(2 \pi)^{2}} \frac{1}{2 E_{k}} \mathrm{e}^{-i E_{k}|t|+i \mathbf{k} \cdot \mathbf{R}},
$$

and $E_{k}=\sqrt{v^{2} k^{2}+m^{2}}$.

Going from real to imaginary time, instead of Equations (4) and (5) we obtain respectively

$$
G(R, \tau)=\left[\partial_{\tau}+i v \gamma \cdot \partial+\gamma^{0} m\right] I(R, \tau),
$$

and

$$
I(R, \tau)=\int \frac{\mathrm{d}^{2} k}{(2 \pi)^{2}} \frac{1}{2 E_{k}} \mathrm{e}^{-E_{k}|\tau|+i k \cdot R}
$$

Performing in Equation (7) angular integration we obtain

$$
I(R, \tau)=\frac{1}{4 \pi} \int_{0}^{\infty} k \frac{\mathrm{e}^{-E_{k}|\tau|}}{E_{k}} J_{0}(k R) \mathrm{d} k
$$

( $J_{0}$ is the Bessel function of zero order). Using mathematical identity [22]

$$
\int_{0}^{\infty} x \frac{\mathrm{e}^{-p \sqrt{x^{2}+z^{2}}}}{\sqrt{x^{2}+z^{2}}} J_{0}(c x) \mathrm{d} x=\frac{\mathrm{e}^{-z \sqrt{p^{2}+c^{2}}}}{\left(p^{2}+c^{2}\right)^{1 / 2}},
$$

we obtain

$$
I(R ; \tau)=\frac{1}{4 \pi v} \frac{\mathrm{e}^{-\sqrt{v^{2} \tau^{2}+R^{2}} \mid m / v}}{\left(v^{2} \tau^{2}+R^{2}\right)^{1 / 2}} .
$$

In the gapless case $m R / v \square 1$ we get [6]

$$
G(R ; \tau)=-\frac{1}{4 \pi} \frac{v \tau+i \gamma \cdot \mathbf{R}}{\left(v^{2} \tau^{2}+R^{2}\right)^{3 / 2}} .
$$

Taking into account that $\left\{\gamma^{i}, \gamma^{j}\right\}=-2 \delta_{i j} \quad(i=1,2)$, and performing integration in Equation (2) we obtain [5]

$$
\chi^{C C}(R)=\frac{1}{64 \pi v R^{3}}, \quad \chi^{A B}(R)=-\frac{3}{64 \pi v R^{3}},
$$

( $C C$ can mean either $A A$ or $B B$ ).

In the opposite case $m R / v \square 1$ we may approximate the GF as

$$
G(R ; \tau)=\frac{m \gamma^{0} R-i|m| \gamma \cdot \mathbf{R}}{4 \pi v R^{2}} \mathrm{e}^{-R|m| / v-v \tau^{2}|m| / 2 R} .
$$

Performing integration in Equation (2), both for intrasublattice, and for inter-sublattice susceptibility we get

$$
\chi=-\frac{1}{8 v}\left(\frac{m}{\pi v R}\right)^{3 / 2} \mathrm{e}^{-2 R|m| / v} .
$$

It is worth paying attention to the fact that Equation
(14) seems to contradict rigorously proved theorem stating that for any bipartite lattice at half filling, the RKKY interaction is antiferromagnetic between impurities sitting on top of atoms belonging to opposite sublattices (i.e., A and B sublattices in graphene), and is ferromagnetic between impurities sitting on top of atoms belonging to the same sublattice $[1,5,23]$. However, the theorem is not applicable to Hamiltonian corresponding to Equation (3), with its last term meaning that if we rewrite the Hamiltonian in the tight-binding representation, the intra-sublattice hopping will appear; hence the lattice is no longer bipartite. More specifically, the spectrum still has the symmetry of that in bipartite lattice, but the wave functions do not [1].

\section{Imaginary Frequency Representation}

The approach will be based on equation [24]

$$
\chi(R)=-\frac{1}{\pi} \int_{-\infty}^{\infty} G^{2}(R ; \omega) \mathrm{d} \omega,
$$

where $G(R ; \omega)$ is the GF in the coordinate-imaginary frequency representation.

\subsection{Gapped Graphene}

The Green's function is

$$
G(\mathbf{k}, \omega)=\frac{-i \omega-v \boldsymbol{\gamma} \cdot \mathbf{k}-\gamma^{0} m}{\omega^{2}+m^{2}+v^{2} k^{2}} .
$$

Hence for the diagonal part of the GF we obtain

$$
\begin{aligned}
G^{(\mathrm{d})}(R, \omega) & =\frac{-i \omega-\gamma^{0} m}{2 \pi} \int_{0}^{\infty} \frac{k J_{0}(k R) \mathrm{d} k}{\omega^{2}+m^{2}+v^{2} k^{2}} \\
& =\frac{-i \omega-\gamma^{0} m}{2 \pi v^{2}} K_{0}\left(\sqrt{\omega^{2}+m^{2}} R / v\right),
\end{aligned}
$$

and for the non-diagonal part

$$
\begin{aligned}
G^{A B}(R, \omega) & =\frac{-v}{2 \pi} \int_{0}^{\infty} \frac{k^{2} J_{1}(k R) \mathrm{d} k}{\omega^{2}+m^{2}+v^{2} k^{2}} \\
& =\frac{-\sqrt{\omega^{2}+m^{2}}}{2 \pi v^{2}} K_{1}\left(\sqrt{\omega^{2}+m^{2}} R / v\right) .
\end{aligned}
$$

Substituting into Equation (15) we obtain [25]

$$
\begin{aligned}
& \chi^{C C}(R)=\frac{1}{4 \pi^{3} v^{4}} \\
& \int_{-\infty}^{\infty}\left(\omega^{2}-m^{2}\right) K_{0}^{2}\left(\sqrt{\omega^{2}+m^{2}} R / v\right) \mathrm{d} \omega . \\
& \chi^{A B}(R)=-\frac{1}{4 \pi^{3} v^{4}} \\
& \int_{-\infty}^{\infty}\left(\omega^{2}+m^{2}\right) K_{1}^{2}\left(\sqrt{\omega^{2}+m^{2}} R / v\right) \mathrm{d} \omega .
\end{aligned}
$$

For $m R / v \square 1$, using mathematical identity [22] 


$$
\begin{aligned}
& \int_{0}^{\infty} x^{\alpha-1} K_{\mu}(c x) K_{\nu}(c x) \mathrm{d} x \\
&=\frac{2^{\alpha-3}}{c^{\alpha} \Gamma(\alpha)} \Gamma\left(\frac{\alpha+\mu+v}{2}\right) \\
& \Gamma\left(\frac{\alpha+\mu-v}{2}\right) \Gamma\left(\frac{\alpha-\mu+v}{2}\right) \Gamma\left(\frac{\alpha-\mu-v}{2}\right),
\end{aligned}
$$

we recover Equation (12).

For $m R / v \square 1$ we may use asymptotic expression for modified Bessel functions

$$
K_{v}(z) \sim \sqrt{\frac{\pi}{2 z}} \mathrm{e}^{-z},
$$

After calculating the resulting integrals in Equation (20) using the Laplace method, we recover Equation (14).

\subsection{Doped Graphene}

For the case of doped (ungapped) graphene the GF is

$$
G(k, \omega)=\frac{1}{i \omega+\mu-v \gamma \cdot \mathbf{k}},
$$

where $\mu$ is the chemical potential. For the diagonal part of the GF in coordinate representation we obtain

$$
\begin{aligned}
& G^{C C}(R, \omega) \\
& =\frac{-i(\omega-i \mu)}{(2 \pi)^{2}} \int_{0}^{\infty} \frac{k \mathrm{~d} k}{(\omega-i \mu)^{2}+v^{2} k^{2}} \cdot \int_{0}^{2 \pi} \mathrm{e}^{i k R \cos \theta} \mathrm{d} \theta \\
& =\frac{-i(\omega-i \mu)}{2 \pi} \int_{0}^{\infty} \frac{k J_{0}(k R) \mathrm{d} k}{(\omega-i \mu)^{2}+v^{2} k^{2}} \\
& =\frac{-i(\omega-i \mu)}{2 \pi v^{2}} K_{0}[\operatorname{sign}(\omega)(\omega-i \mu) R / v],
\end{aligned}
$$

and for the non-diagonal part

$$
\begin{aligned}
& G^{A B}(R, \omega) \\
& =\frac{-v}{(2 \pi)^{2}} \int_{0}^{\infty} \frac{k^{2} \mathrm{~d} k}{(\omega-i \mu)^{2}+v^{2} k^{2}} \cdot \int_{0}^{2 \pi} \mathrm{e}^{i k R \cos \theta+i \theta} \mathrm{d} \theta \\
& =\frac{-v}{2 \pi} \int_{0}^{\infty} \frac{k^{2} J_{1}(k R) \mathrm{d} k}{(\omega-i \mu)^{2}+v^{2} k^{2}} \\
& =\frac{-\operatorname{sign}(\omega)(\omega-i \mu)}{2 \pi v^{2}} K_{1}[\operatorname{sign}(\omega)(\omega-i \mu) R / v] .
\end{aligned}
$$

$K_{0}$ and $K_{1}$ are the modified Bessel function of zero and first order respectively. We have used mathematical identity, valid for $\operatorname{Re} z>0 \quad$ [22]

$$
\int_{0}^{\infty} \frac{x^{v+1}}{\left(x^{2}+z^{2}\right)^{\rho}} J_{v}(c x) \mathrm{d} x=\frac{c^{\rho-1} z^{v-\rho+1}}{\left.2^{\rho-1} \Gamma(\rho)\right)} K_{v-\rho+1}(c z)
$$

The susceptibility (15) is expressed through the integrals

$$
\operatorname{Re}\left\{\int_{0}^{\infty}(\omega-i \mu)^{2} K_{0,1}^{2}[(\omega-i \mu) R / v] \mathrm{d} \omega\right\}
$$

Considering integrals in the complex plane it is convenient to deform the contour of integration and present the integrals as

$$
\operatorname{Re}\left\{\int_{0}^{\infty} \omega^{2} K_{0,1}^{2}(\omega R / v)+\int_{-i \mu}^{0} \omega^{2} K_{0,1}^{2}(\omega R / v) \mathrm{d} \omega\right\} .
$$

Taking into account the identity

$$
K_{\alpha}(-i x)=\frac{\pi}{2} i^{\alpha+1}\left[J_{\alpha}(x)+i Y_{\alpha}(x)\right],
$$

we get [12]

$$
\begin{aligned}
& \chi_{\mu}^{C C}(R)=\chi_{\mu=0}^{C C}(R)\left[1-16 \int_{0}^{k_{F} R} \mathrm{dzz}^{2} J_{0}(z) Y_{0}(z)\right] \\
& \chi_{\mu}^{A B}(R)=\chi_{\mu=0}^{A B}(R)\left[1+\frac{16}{3} \int_{0}^{k_{F} R} \mathrm{dzz}^{2} J_{1}(z) Y_{1}(z)\right]
\end{aligned}
$$

where $k_{F}=\mu / v$, and $\chi_{\mu=0}(R)$ are given by Equation (12). The integrals in Equation (30) can be presented in terms of Meijer functions [12,19] (I address the reader to these References for the details).

It is interesting to compare the RKKY exchange in doped graphene, with its two sublattices and linear dispersion law, with that in ordinary two-dimensional electron gas. For the latter the Green's function is

$$
G(k, \omega)=\frac{1}{i \omega+\mu-k^{2} / 2 m},
$$

and the susceptibility turns out to be [26]

$$
\chi(R) \sim \frac{1}{R^{2}} \int_{0}^{k_{F} R} \mathrm{dzz} J_{0}(z) Y_{0}(z) .
$$

\section{Conclusion}

In the end we would like to mention again that in the case considered, the GF calculations in Euclidean space, as it is not infrequently happens, have advantages over those in Minkowskii space. In particular, using the former one have to operate only with the convergent integrals, in distinction to what happens when one uses the latter.

\section{Acknowledgements}

Discussions with G. Gumbs, S. Kettemann, D. Loss, M. Sherafati, K. Szalowski, and K. Ziegler were very illuminating for the author.

\section{REFERENCES}

[1] E. Kogan, "RKKY Interaction in Graphene," Physical Review B, Vol. 84, No. 11, 2011, Article ID: 115119. doi:10.1103/PhysRevB.84.115119

[2] M. A. H. Vozmediano, M. P. Lopez-Sancho, T. Stauber 
and F. Guinea, "Local Defects and Ferromagnetism in Graphene Layers," Physical Review B, Vol. 72, No. 15, 2005, Article ID: 155121. doi:10.1103/PhysRevB.72.155121

[3] V. K. Dugaev, V. I. Litvinov and J. Barnas, "Exchange Interaction of Magnetic Impurities in Graphene," Physical Review B, Vol. 74, No. 22, 2006, Article ID: 224438. doi:10.1103/PhysRevB.74.224438

[4] L. Brey, H. A. Fertig and S. D. Sarma, "Diluted Graphene Antiferromagnet," Physical Review Letters, Vol. 99, No. 11, 2007, Article ID: 116802. doi:10.1103/PhysRevLett.99.116802

[5] S. Saremi, "RKKY in Half-Filled Bipartite Lattices: Graphene as an Example," Physical Review B, Vol. 76, No. 18, 2007, Article ID: 184430.

[6] V. V. Cheianov, O. Syljuasen, B. L. Altshuler and V. Fal'ko, "Ordered States of Adatoms on Graphene," Physical Review B, Vol. 80, No. 23, 2009, Article ID: 233409. doi:10.1103/PhysRevB.80.233409

[7] A. M. Black-Schaffer, "RKKY Coupling in Graphene," Physical Review B, Vol. 81, No. 20, 2010, Article ID: 205 416. doi:10.1103/PhysRevB.81.205416

[8] M. Sherafati and S. Satpathy, "RKKY Interaction in Graphene from the Lattice Green's function," Physical Review B, Vol. 83, No. 16, 2011, Article ID: 165425. doi:10.1103/PhysRevB.83.165425

[9] B. Uchoa, T. G. Rappoport and A. H. C. Neto, "Kondo Quantum Criticality of Magnetic Adatoms in Graphene," Physical Review Letters, Vol. 106, No. 1, 2011, Article ID: 016801. doi:10.1103/PhysRevLett.106.016801

[10] J. E. Bunder and H.-H. Lin, "Ruderman-Kittel-KasuyaYosida Interactions on a Bipartite Lattice," Physical Review B, Vol. 80, No. 15, 2009, Article ID: 153414. doi:10.1103/PhysRevB.80.153414

[11] S. R. Power and M. S. Ferreira, "Electronic Structure of Graphene beyond the Linear Dispersion Regime," Physical Review B, Vol. 83, No. 15, 2011, Article ID: 155432. doi:10.1103/PhysRevB.83.155432

[12] M. Sherafati and S. Satpathy, "Analytical Expression for the RKKY Interaction in Doped Graphene," Physical Review $B$, Vol. 84, No. 12, 2011, Article ID: 125416. doi:10.1103/PhysRevB.84.125416

[13] K. Szalowski, "RKKY Coupling between Impurity Spins in Graphene Nanoflakes," Physical Review B, Vol. 84, No. 20, 2011, Article ID: 205409. doi:10.1103/PhysRevB.84.205409

[14] J. E. Bunder and J. M. Hill, "Geometric Influence on Ruderman-Kittel-Kasuya-Yosida Interactions in Zigzag Carbon Nanotubes," Journal of Chemical Physics, Vol. 136, No. 15, 2012, Article ID: 154504. doi:10.1063/1.4704677

[15] H. Lee, J. Kim, E. R. Mucciolo, G. Bouzerar and S. Kettemann, "RKKY Interaction in Disordered Graphene," Physical Review B, Vol. 85, No. 7, 2012, Article ID: 075420. doi:10.1103/PhysRevB.85.075420

[16] M. Sherafati and S. Satpathy, "On the Ruderman-KittelKasuya-Yosida Interaction in Graphene," Proceedings of the International Workshop on Functional Materials, Berhampur, 20-22 December 2011, pp. 24-33. doi:10.1063/1.4736868

[17] L. Jiang, X. Lu, W. Gao, G. Yu, Z. Liu and Y. Zheng, "RKKY Interaction in AB-Stacked Multilayer Graphene," Journal of Physics: Condensed Matter, Vol. 24, No. 20, 2012, Article ID: 206003.

[18] J. Klinovaja and D. Loss, "RKKY Interaction in Carbon Nanotubes and Graphene Nanoribbons." arXiv:1211.3067v1.

[19] O. Roslyak, G. Gumbs and D. Huang, "Gap-Modulated Doping Effects on Indirect Exchange Interaction between Magnetic Impurities in Graphene.” arXiv:1211.5099v1.

[20] M. I. Katsnelson, "Graphene: Carbon in Two Dimensions," Cambridge University Press, Cambridge, 2012. doi:10.1017/CBO9781139031080

[21] R. P. Feynman, "Quantum Electrodynamics," Westview Press, 1961.

[22] A. P. Prudnikov, Yu. A. Brychkov and O. I. Marichev, "Integrals and Series," Gordon and Breach Science Publishers, 1986.

[23] V. M. Pereira, J. M. B. Lopes dos Santos and A. H. C. Neto, " Modeling Disorder in Graphene," Physical Review B, Vol. 77, No. 11, 2008, Article ID: 115109. doi:10.1103/PhysRevB.77.115109

[24] A. A. Abrikosov, L. P. Gorkov and I. E. Dzyloshinski, "Methods of Quantum Field Theory in Statistical Physics," Pergamon Press, 1965.

[25] V. K. Dugaev, V. I. Litvinov and P. P. Petrov, "Magnetic Impurity Interactions in a Quantum Well on the Base of IV-VI Semiconductors," Superlattices and Microstructures, Vol. 16, No. 4, 1994, pp. 413-417.

[26] B. Fischer and M. W. Klein, "Magnetic and Nonmagnetic Impurities in Two-Dimensional Metals," Physical Review B, Vol. 11 , No. 5 , 1975 , pp. 2025-2029. doi:10.1103/PhysRevB.11.2025

[27] V. I. Litvinov and V. K. Dugaev, "RKKY Interaction in One- and Two-Dimensional Electron Gases," Physical Review B, Vol. 58, No. 7, 1998, pp. 3584-3585. doi:10.1103/PhysRevB.58.3584 


\section{Appendix: Approaches Based on Real Frequency GF}

The approach, used in $[2,4,5]$ is based on equation

$$
\chi(R)=\int \frac{\mathrm{d}^{2} \mathbf{q}}{(2 \pi)^{2}} \chi(\omega=0, \mathbf{q}) \mathrm{e}^{i \mathbf{q} \cdot \mathbf{R}},
$$

where

$$
\chi(\omega=0, \mathbf{q})=2 \int \frac{\mathrm{d}^{2} \mathbf{k}}{(2 \pi)^{2}} \frac{n_{F}\left(\xi_{\mathbf{k}}\right)-n_{F}\left(\xi_{\mathbf{k}+\mathbf{q}}\right)}{E_{\mathbf{k}+\mathbf{q}}-E_{\mathbf{k}}} ;
$$

$\xi_{n}=E_{n}-\mu$ and $n_{F}(\xi)=\left(\mathrm{e}^{\beta \xi}+1\right)^{-1}$ is the Fermi distribution function. This approach, though looking quite straightforward, brings with it a problem. In a model of infinite Dirac cones, for $\chi(\omega=0, \mathbf{q})$ we obtain a diverging integral.

The problem can be formulated in a different way. Being calculated in a realistic band model, with the bands of finite width, $\chi(\omega=0, \mathbf{q})$ is not a universal quantity. It depends not only on infrared physics, but on the properties of electron spectrum and eigenfunctions in the whole Brillouin zone (even for small q).

Another approach, formulated in [6], starts from a well-known equation for the susceptibility

$$
\chi(R)=\frac{2 i}{\pi} \int_{-\infty}^{\infty} G^{2}(R, E) \mathrm{d} E
$$

where $G$ is the retarded green's function. Here again the integral diverges on both limits of integration. How- ever the authors changed the contour of integration, transforming the divergent integral (5) into the convergent integral along the imaginary axis (see also [27]). The authors also considered RKKY interaction in gapped graphene, when the power law decrease of the interaction with the distance turns into the exponential law. Actually, the authors made the transition from real to imaginary frequencies, so some of our results are close to ones obtained in [6].

The approach, using formula

$$
\chi\left(r, r^{\prime}\right)=\delta n(r) / V\left(r^{\prime}\right)
$$

and, hence, calculating electron susceptibility on the basis of equation

$$
\chi(R)=-\frac{2}{\pi} \int_{-\infty}^{E_{F}} \operatorname{Im}\left[G^{2}(R, E)\right] \mathrm{d} E
$$

where $E_{F}$ is the Fermi energy, was first used, in application to graphene to the best of our knowledge, in [8]. An advantage of this approach is that it allows to easily consider the case of doped graphene, the disadvantage is that the approach, like the one presented above, has to deal with the divergent integral (the integral with respect to $\mathrm{d} E$ diverges at the lower limit of integration). To obtain finite values from these divergent integral, similar to what was necessary for the approach mentioned in the beginning of the, one has to implement the complicated (and to some extent arbitrary) cut-off procedure [5]. 